\title{
Analysis of the specific vibration modes of goethite (a-FeOOH) by terahertz spectroscopy and calculations of the vibration frequencies of a single molecule using density functional theory
}

\author{
Ryo Hasegawa ${ }^{1}$, Takashi Kimura ${ }^{1}$, Tadao Tanabe ${ }^{1}$, Katsuhiro Nishihara ${ }^{2}$, Akira Taniyama ${ }^{2}$, \\ Yutaka Oyama' \\ ${ }^{1}$ Dep. Materials Science and Engineering, Tohoku University, Sendai, Japan \\ ${ }^{2}$ Nippon Steel \& Sumitomo Metal Co., Ltd. Amagasaki, Japan \\ Correspondence: Tadao Tanabe. E-mail: tadao.tanabe.b1@tohoku.ac.jp
}

Received: February 2, 2018

Accepted: February 12, 2018

Online Published: February 18, 2018

DOI: $10.5430 / j b g c . v 8 n 1 p 29$

URL: http://dx.doi.org/10.5430/jbgc.v8n1p29

\begin{abstract}
Steel sheet with an insulator to prevent corrosion is used for various purposes including in building and car manufacture. Terahertz waves, for which insulators are highly permeable and metal surfaces are highly reflective, have been studied in order to establish a new inspection technology for these steel plates. In our previous research, spectroscopic measurements in the 1.0-4.0 THz range, generated by a GaP crystal, were carried out in order to collect information on the infrared activity of the metal corrosion products formed on $\mathrm{Zn}$-Al hot-dip galvanized steel sheet. In the previous work, the infrared activity of Fe-based corrosion products was not examined. To examine these products, we conducted $\mathrm{THz}$ spectroscopy on goethite $(\alpha-\mathrm{FeOOH})$ in the range from 8.4 to $11.0 \mathrm{THz}$, generated by a GaSe crystal. The results of Attenuated Total Reflectance (ATR) FTIR spectral measurements and molecular vibration calculations were analyzed, on the basis of which the natural vibration modes of $\alpha-\mathrm{FeOOH}$ in the $\mathrm{THz}$ frequency range were assigned.
\end{abstract}

\section{Key Words:}

Terahertz, Non-destructive inspection, Corrosion, Goethite, $\alpha-\mathrm{FeOOH}$, Density functional theory

\section{Introduction}

Terahertz waves have a number of useful characteristics. First, they are highly permeable in non-polarized materials; second, they are highly reflective at metal surfaces; third, they can be used to obtain finger print spectra for molecular bonding; and fourth, they are safe to use as they are harmless to human tissue. THz spectroscopy can be used to inspect the condition of steel substrates covered by an insulator/coating. Metal corrosion products can be qualitatively and quantitatively measured through the absorption of $\mathrm{THz}$ waves at the natural vibration frequencies of the molecules in these products. In addition, $\mathrm{THz}$ spectroscopy has the advantage of having high resolution, and there is no need for a large interferometer or a long optical path such as needed for Fourier Transform Infrared Spectroscopy (FTIR) spectroscopy. However, practical THz spectroscopy has yet to be demonstrated. In the 1990s, THz spectroscopy was conducted using $\mathrm{THz}$ waves generated using a photoconductive antenna ${ }^{[1]}$. It has since become possible to make measurements over a wide range of tunable frequencies using difference frequency generation (DFG) crystals, which have high nonlinear optical effects. Thus, we have devoted much attention to studying this phenomenon ${ }^{[2-4]}$. As THz spectroscopy has developed, the infrared activities of many organic and biological substances have been reported ${ }^{[5-10]}$; however, the infrared activity of 
Fe-based metal corrosion products has not yet been reported ${ }^{[11]}$. The production mechanism for Fe-based corrosion products is shown in Fig. $1^{[12]}$. When $\mathrm{Fe}^{3+}$ ions are generated in steel sheet, they aggregate in neutral or basic solutions to form colloids of $\mathrm{Fe}(\mathrm{OH})_{\mathrm{x}}^{3-\mathrm{x}}$. If the oxidation rate is fast, the $\mathrm{Fe}^{3+}$ ion deprotonates into $\mathrm{Fe}(\mathrm{OH})_{3}$. When $\mathrm{Fe}(\mathrm{OH})_{3}$ is hydrolyzed, $\alpha-\mathrm{FeOOH}$ is formed. In contrast, if the oxidation rate is slow, green rust, which is a mixture of $\mathrm{Fe}(\mathrm{OH})_{2}$ and $\mathrm{Fe}(\mathrm{OH})_{3}$ is produced. When the green rust is completely oxidized, it changes to $\alpha-\mathrm{FeOOH}{ }^{[13-14]}$. The qualitative and quantitative evaluation of $\alpha-\mathrm{FeOOH}$ is an important factor that can be used to estimate the condition of the steel substrate. Our research group has performed spectroscopic analysis on $\alpha-\mathrm{FeOOH}$ at frequencies of 1.0-4.0 THz, which is the range of $\mathrm{THz}$ waves generated by GaP crystals. The obtained spectrum is shown in Fig.2. However, the infrared activity of $\alpha$-FeOOH was not obtained in this frequency range. Therefore in this study, spectroscopic analysis of $\alpha-\mathrm{FeOOH}$ was carried out using a GaSe crystal, which can generate $\mathrm{THz}$ waves in a higher frequency region than GaP crystals. Calculations of the vibration frequencies of single molecules were conducted enabling us to assign the natural vibration modes of $\alpha-\mathrm{FeOOH}$.

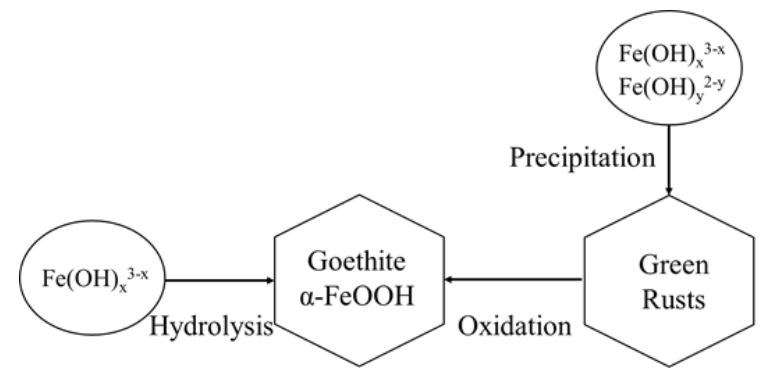

Figure 1. Mechanism for the generation of Fe-based corrosion products ${ }^{[12]}$.

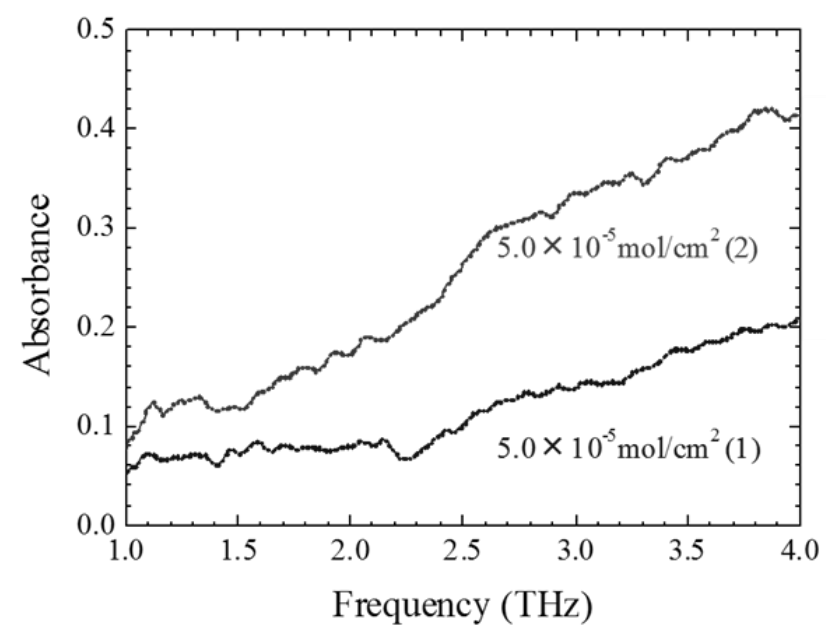

Figure 2. Absorption spectra of $\alpha-\mathrm{FeOOH}$ at $1.0-4.5 \mathrm{THz}$.

\section{Experiment}

\subsection{Samples}

For the $\mathrm{THz}$ transmittance measurements we prepared pellets consisting of a mixture of standard $\alpha$-FeOOH powder and polyethylene powder. The two powders were ground by mortar and pestle for $10 \mathrm{mins}$. Pellets with different mixture ratios were prepared, with concentrations of 1.0, 3.0, $5.0 \mathrm{wt} \%$ of $\alpha-\mathrm{FeOOH}$. The diameter and thickness of the pellets were 20 $\mathrm{mm}$ and less than $1 \mathrm{~mm}$, respectively, and were compressed at a pressure of $1.0 \mathrm{ton} / \mathrm{cm}^{2}$ for $1 \mathrm{~min}$. During compression, the pellets were tilted $2^{\circ}$ to prevent interference of THz wave due to the pellet thickness. Details of the $\alpha$-FeOOH powder used in the pellets is shown in Table 1. 
Table 1. Details of $\alpha-\mathrm{FeOOH}$ powder.

\begin{tabular}{lll}
\hline & Molecular Weight & Purity \\
\hline$\alpha-\mathrm{FeOOH}$ & 88.85 & $>99 \%$ \\
\hline
\end{tabular}

\subsection{Optical System}

The experimental set up for the $\mathrm{THz}$ spectrometer has been described in previous reports ${ }^{[15-16]}$. THz transmittance spectroscopy was carried out as shown in Fig.3. The probe beam is produced by a frequency variable Cr:Forsterite laser excited by a Nd:YAG laser. Two different frequencies close to the infrared region are introduced into the GaSe crystal. The THz waves generated are directed toward the sample pellet and detected by a $4.2 \mathrm{~K} \mathrm{Si}$ bolometer. The parameters of the optical stage for each frequency are controlled by a computer. The frequency range of the THz waves generated from the GaSe crystal is from 8.4 to $11.0 \mathrm{THz}$. THz radiation generated in the GaP crystal obtained in our previous study and in the GaSe crystal in this study is shown in Fig.4. It can be seen from the figure that generating terahertz radiation in the high frequency region is possible using a GaSe crystal. The linewidth of the $\mathrm{THz}$ radiation is $500 \mathrm{MHz}$.

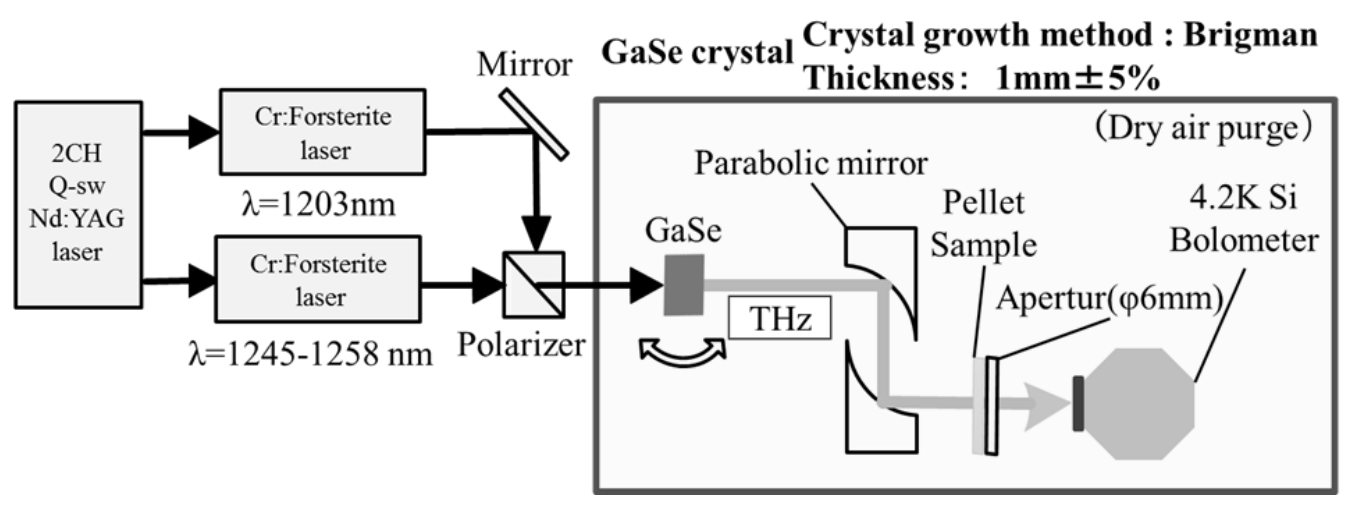

Figure 3. Schematic diagram for the THz spectroscopic measurements.
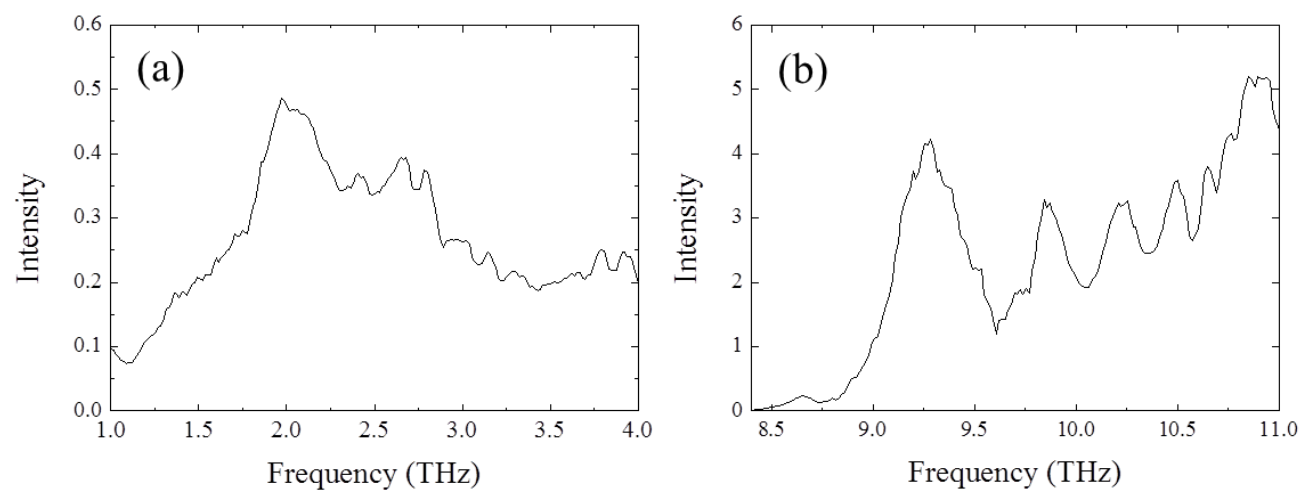

Figure 4. $\mathrm{THz}$ radiation in the frequency range 1.0-4.0 $\mathrm{THz}$ generated in the GaP crystal (a), and in the frequency range 8.4-11.0 THz generated in the GaSe crystal (b).

\subsection{ATR-FTIR Spectroscopy}

ATR-FTIR spectroscopic measurements of the standard $\alpha$-FeOOH specimen were conducted. ATR-FTIR spectroscopy is an analytical method that uses the absorption spectrum in the infrared region for qualitatively and quantitatively evaluating substances. The measurements were carried out in the range of $100 \mathrm{~cm}^{-1}-4000 \mathrm{~cm}^{-1}(3.0-12.0 \mathrm{THz})$ in the far-infrared region at room temperature. For these measurements, we used a Thermo Scientific ${ }^{\mathrm{TM}}$ Nicolet $^{\mathrm{TM}}$ iS $^{\mathrm{TM}} 50$ FTIR spectrometer with a built-in ATR. The measurement step was $8 \mathrm{~cm}^{-1}$ and the $S / N$ ratio was 55,000 . From the measurements, three broad peaks were observed as shown in Fig.5. Three broad absorption peaks were obtained at (1)9.95 THz, (2)10.54 THz and (3) $11.38 \mathrm{THz}$ by ATR-FTIR spectroscopy. However, these peaks are considered to be the combination of several 
absorption peaks of which the full width at half maximums (FWHM) are too large to enable them to be assigned to any particular molecular vibration mode. Since high resolution can be obtained with terahertz spectroscopy, this can help us clarify the absorption peaks and assign them in this case.

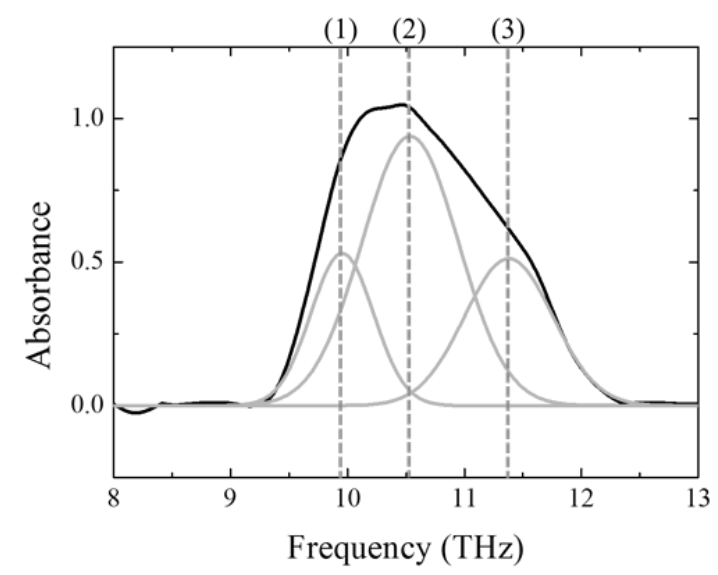

Figure 5. ATR-FTIR spectroscopy of $\alpha-\mathrm{FeOOH}$.

\subsection{DFT Calculations}

By comparing the experimental results with calculations of the vibration frequencies in single molecules, it is possible to assign the intramolecular vibration modes. Single molecule vibrations for $\alpha-\mathrm{FeOOH}$ were calculated using density functional theory (DFT) with the aid of the Gaussian $09^{\circledR}$ program package. DFT is a quantum chemical method and is known to be a reliable method for obtaining vibration frequencies. Due to the different possible spin states of iron and oxygen atoms, the calculations were performed using an unrestricted spin B3LYP hybrid density functional and the 6-31G $(\mathrm{d}, \mathrm{p})$ basis set which is the split valence double zeta basis set.

\section{Results and Discussion}

THz spectroscopic measurements were conducted, and the results are summarized in Fig.6. Four absorption peaks were observed at (1) $8.9 \mathrm{THz},(2) 10.0 \mathrm{THz},(3) 10.2 \mathrm{THz}$ and (4)10.3 THz (Fig.6 a). These absorption peaks were fitted to Gaussian functions and a quantitative relationship between the peaks was obtained (Fig. 6 b). From the results of the calculations using DFT, two natural vibration modes, at $8.61 \mathrm{THz}$ and $9.23 \mathrm{THz}$, were identified. The vibration mode at 8.61 THz is the bending vibration between oxygen and iron atoms and the absorption peak at $9.23 \mathrm{THz}$ is the bending vibration between oxygen and hydrogen atoms. The dependence of the $\mathrm{THz}$ absorption area for the various $\alpha-\mathrm{FeOOH}$ mixture ratios can be divided into 2 groups, one in which the gradient is $>1.0$, the other in which the gradient is $<1.0$.
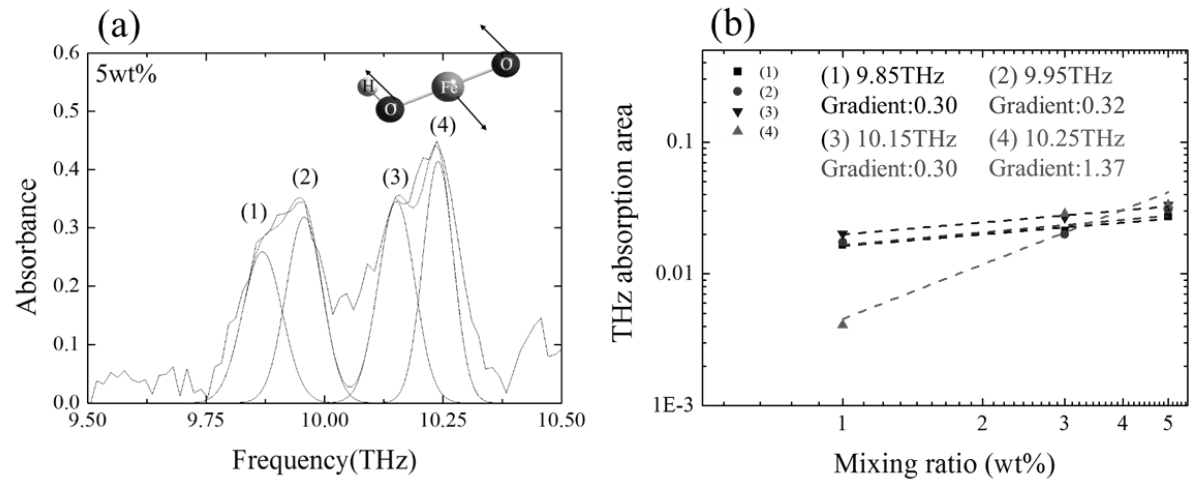

Figure 6. Absorption peaks in the THz spectrum (a), the dependence of the integrated intensity of

$\mathrm{THz}$ absorption with respect to mixing ratio (b). 
These are expected to be for the different specific vibration modes of $\alpha$-FeOOH. The intramolecular vibrations become more active as the mixture ratio increases. Conversely, the intermolecular vibrations become more restrained as the mixture ratio increases, due to the Mass Action Law (MAL). Assume that $\mathrm{n}$ is an intermolecular bond between molecules M1 and M2. $\mathrm{n}$ is, for example, a hydrogen bond or a hydrated bond. Furthermore, consider that the integrated intensity of the absorption band of a specific frequency appearing in the $\mathrm{THz}$ spectrum is proportional to the number of intermolecular bonds. Then, when M1 and M2 are bound with two intermolecular bonds, the equilibrium chemical reaction formula is

$$
\mathrm{M} 1+2 \mathrm{n}+\mathrm{M} 2 \leftrightarrows(\mathrm{M} 1-2 \mathrm{n}-\mathrm{M} 2)
$$

From the MAL, the equilibrium reaction constant $\mathrm{K}$ is

$$
\mathrm{K}=\frac{[\mathrm{M} 1][\mathrm{n}]^{2}[\mathrm{M} 2]}{[\mathrm{M} 1-2 \mathrm{n}-\mathrm{M} 2]}
$$

Therefore, the intermolecular bond concentration $[\mathrm{n}]$ is

$$
[\mathrm{n}]=\left(\mathrm{K} \frac{[\mathrm{M} 1-2 \mathrm{n}-\mathrm{M} 2]}{[\mathrm{M} 1][\mathrm{M} 2]}\right)^{1 / 2}
$$

This represents a bimolecular vibration, and the integrated absorption band intensity is proportional to the square root of the concentration. Generally, if there are $\mathrm{x}$ loosely bonded intermolecular bonds, the equilibrium chemical reaction formula is

$$
\mathrm{M} 1+\mathrm{xn}+\mathrm{M} 2 \leftrightarrows(\mathrm{M} 1-\mathrm{xn}-\mathrm{M} 2)
$$

The equilibrium reaction constant $\mathrm{K}$ and intermolecular bond concentration $[\mathrm{n}]$ are

$$
\begin{gathered}
\mathrm{K}=\frac{[\mathrm{M} 1][\mathrm{n}]^{\mathrm{x}}[\mathrm{M} 2]}{[\mathrm{M} 1-\mathrm{xn}-\mathrm{M} 2]} \\
{[\mathrm{n}]=\left(\mathrm{K} \frac{[\mathrm{M} 1-\mathrm{xn}-\mathrm{M} 2]}{[\mathrm{M} 1][\mathrm{M} 2]}\right)^{1 / \mathrm{x}}}
\end{gathered}
$$

Thus, it can be considered that the integral absorption band intensity is proportional to the $1 / \mathrm{x}$ the power of the concentration. For example, in the case of bimolecular vibrations, it is proportional to the square root of the concentration and the gradient is 0.5 . And In the case of tri-molecular vibrations, it is proportional to the cube root of the concentration, and the gradient is 0.33 . Therefore absorption peak (4), which has a quantitative relationship (Gradient $>1.0$ ), is expected to be an intramolecular vibration. On the other hand, the remaining absorption peaks, (1), (2) and (3), which have quantitative

\begin{tabular}{|c|c|c|}
\hline $\begin{array}{l}\text { Powder sample by ATR-FTIR } \\
\text { spectroscopy }(\mathrm{THz})\end{array}$ & $\begin{array}{l}\text { Compressed Pellet by } \mathrm{THz} \text { spectroscopy } \\
(\mathrm{THz})\end{array}$ & $\begin{array}{l}\text { Single molecular vibration calculation by } \\
\text { DFT }(\mathrm{THz})\end{array}$ \\
\hline \multirow{3}{*}{9.95} & 9.85 (Gradient:0.30) & \\
\hline & 9.95 (Gradient:0.32) & \\
\hline & 10.15 (Gradient:0.30) & \\
\hline 10.54 & 10.25 & 8.61 \\
\hline 11.38 & & 9.23 \\
\hline
\end{tabular}
relationships (Gradient $\fallingdotseq 0.30$ ), are expected to be intermolecular vibrations. The ATR-FTIR spectroscopic measurements, $\mathrm{THz}$ spectroscopic measurements and the calculations of single molecule vibrations using DFT are summarized in the Table 2.

Table 2. Summary of the results of ATR-FTIR spectroscopic measurements, THz spectroscopic measurements, and calculations of single molecule vibrations using DFT.

\section{Conclusion}

In this paper, the absorption characteristics of $\alpha-\mathrm{FeOOH}$ are analyzed by ATR-FTIR spectroscopy, THz spectroscopy and calculations of single molecule vibrations using DFT. From the results, the absorption peak observed at $10.25 \mathrm{THz}$ is expected to be due to intramolecular vibrations. Conversely, the remaining absorption peaks, at 9.85, 9.95 and $10.15 \mathrm{THz}$, are expected to be due to intermolecular vibrations. This work demonstrates that higher resolution can be obtained with $\mathrm{THz}$ spectroscopy compared to ATR-FTIR spectroscopy, and that, from the gradient of the THz absorption area with 
respect to mixture ratio and by comparison with the results of calculations of single molecule vibrations using DFT, the vibration mode can be assigned.

\section{Acknowledgement}

This study is partially supported by "Fundamental Research and Human Resources Development Program for Nuclear Decommissioning related to Integrity Management of Critical Structures including Primary Containment Vessel and Reactor Building, and Fuel Debris Processing and Radioactive Waste Disposal" carried out under the Center of World Intelligence Project for Nuclear S\&T and Human Resource Development by the Ministry of Education, Culture, Sports, Science and Technology of Japan.

\section{References}

[1] Grischkowsky, D. R., \& Mittleman, D. (2003). Sensing with Terahertz Radiation: Optical Sciences. Berlin: Spriger-Verlag. 1. https://doi.org/10.1007/978-3-540-45601-8

[2] Tanabe, T., Suto, K., Nishizawa, J., Kimura, T., \& Saito, K. (2003). Frequency-tunable high-power terahertz wave generation from GaP. J. Appl. Phys., 93(8), 4610-5615. https://doi.org/10.1063/1.1560573

[3] Tanabe, T., Suto, K., Nishizawa, J., Saito, K., \& Kimura, T. (2003). Tunable terahertz wave generation in the 3- to 7-THz region from GaP. Appl. Phys. Lett., 83(2), 237-239. https://doi.org/10.1063/1.1592889

[4] Tanabe, T., Suto, K., Nishizawa, J., Saito, K., \& Kimura, T. (2003). Frequency-tunable terahertz wave generation via excitation of phonon- polaritons in GaP. Appl. Phys. D: Appl. Phys., 36(8), 953-957. https://doi.org/10.1088/0022-3727/36/8/302

[5] Hu, B. B., \& Nuss, M. C. (1995). Imaging with terahertz waves. Opt. Lett., 20(16), 1716-1719. https://doi.org/10.1364/OL.20.001716

[6] Mittleman, D. M., Gupta, M., Neelamani, R., Baraniuk, R. G. Rudd, J. V., \& Koch, M. (1999). Recent advances in terahertz imaging. Appl. Phys. B, 68(6), 1085-1096. https://doi.org/10.1007/s003409900011

[7] Ferguson, B., \& Zhang, X. C. (2002). Materials for terahertz science and technology. Nat. Mater., 1, 26-33. https://doi.org/10.1038/nmat708

[8] Kawase, K., Ogawa, Y., Watanabe, Y., \& Inoue, H. (2003). Non-destructive terahertz imaging of illicit drugs using spectral fingerprints. Opt. Express, 11(20), 2549-2554. https://doi.org/10.1364/OE.11.002549

[9] Nishizawa, J. (2004). Development of THz wave oscillation and its application to molecular sciences. Proc. Jpn. Acad. Ser. B, 80(2), 74-81. https://doi.org/10.2183/pjab.80.74

[10] Tonouchi, M. (2007). Cutting-edge terahertz technology. Nat. Photo, 1, 97-105. https://doi.org/10.1038/nphoton.2007.3

[11] Nakamura, Y., Kariya, H., Sato, A., Tanabe, T., Nishihara, K., Taniyama, A., Nakajima, K., Maeda, K., \& Oyama, Y. (2014). Non-destructive Corrosion Diagnosis of Painted Hot-dip Galvanizing Steel Sheets by Using THz Spectral Imaging. Corrosion Engineering, 63(9), 411-416. https://doi.org/10.3323/jcorr.63.504

[12] Schwertmann, U., \& Cornell, R. M. (2000). Iron Oxides in the Laboratory: Preparation and Characterization. Wiley-VCH. p5. https://doi.org/10.1002/9783527613229

[13] Ishikawa, T., Takeuchi, K., Kandori, K., \& Nakayama, T. (2005). Transformation of $\gamma$-FeOOH to $\alpha$-FeOOH in acidic solutions containing metal ions. Colloid Surf. A: Physicochem. Eng. Aspects, 266(1-3), 155-159. https://doi.org/10.1016/j.colsurfa.2005.06.024

[14] Misawa, T. (1973). The thermodynamic consideration for $\mathrm{Fe}-\mathrm{H}_{2} \mathrm{O}$ system at $25{ }^{\circ} \mathrm{C}$. Corrosion Sci., $13(9)$, $659-676$. https://doi.org/10.1016/S0010-938X(73)80037-X

[15] Suto, K., Sasaki, T., Tanabe, T., \& Kimura, T. GaP THz wave generator and THz spectrometer using Cr:Forsterite lasers. Rev. Sci. Instrum, 76(12), 123109. https://doi.org/10.1063/1.2140223

[16] Nishizawa, J., Suto, K., Sasaki, T., Tanabe, T., \& Kimura, T. (2003). Spectral measurement of teraherz vibrations of biomolecules using a GaP terahertz-wave generator with automatic scanning control. J. Phys. D: Apply. Phys., 36(23), $2958-2961$. https://doi.org/10.1088/0022-3727/36/23/015 